\title{
Boundary Entropy Can Increase Under Bulk RG Flow
}

\author{
Daniel Green, ${ }^{*}$ Michael Mulligan, ${ }^{\dagger}$ and David Starr ${ }^{\ddagger}$ \\ SLAC and Department of Physics, Stanford University, Stanford, CA 94305-4060
}

\begin{abstract}
The boundary entropy $\log (g)$ of a critical one-dimensional quantum system (or two-dimensional conformal field theory) is known to decrease under renormalization group (RG) flow of the boundary theory. We study instead the behavior of the boundary entropy as the bulk theory flows between two nearby critical points. We use conformal perturbation theory to calculate the change in $g$ due to a slightly relevant bulk perturbation and find that it has no preferred sign. The boundary entropy $\log (g)$ can therefore increase during appropriate bulk flows. This is demonstrated explicitly in flows between minimal models. We discuss the applications of this result to D-branes in string theory and to impurity problems in condensed matter.
\end{abstract}

*Electronic address: drgreen@stanford.edu

$\dagger$ Electronic address: mcmullig@stanford.edu

¥Electronic address: dbstarr@stanford.edu

Submitted to Journal of Statistical Mechanics

Work supported in part by US Department of Energy contract DE-AC02-76SF00515 


\section{INTRODUCTION}

Two-dimensional quantum field theories play an important role in many different branches of physics, ranging from string theory to condensed matter. It is therefore important to understand the general features and qualitative behavior of these theories. Much is known when the theory is conformally invariant (CFT), due to the large symmetry group. However, less is known about the general features of non-conformal theories. One means of gaining insight is to perturb a given critical theory and follow the resulting trajectory under RG flow. In particular, it is interesting to learn what new critical theory is the endpoint of the RG flow and to compare the properties of the new and old systems.

A famous result in this direction is the Zamolodchikov $c$ theorem [1], which states that the central charge of a CFT always decreases under such a flow. There is an analogous theorem which holds for two-dimensional CFTs with boundary (BCFTs). For these systems Affleck and Ludwig [2] introduced a quantity $g$, known as the generalized "ground-state degeneracy" or "boundary entropy." The $g$ theorem states that this quantity decreases under RG flow of the boundary, so long as the bulk theory remains critical during the boundary flow. This theorem has been proven both perturbatively [3] and non-perturbatively [4]. However, $a$ priori there is no reason for this qualitative behavior of $g$ to persist when the bulk theory also undergoes RG flow.

There are many situations where one is specifically interested in quantities related to the boundary. For example, in string theory the boundary is associated with D-branes, and the brane tension is given by $g$ [5, 6]. The Kondo effect can be understood using a BCFT [3, 7, 8] for which $\log (g)$ is the impurity entropy. These are physical quantities which one might wish to track as the bulk theory moves between two critical points.

In this note we consider the change in $g$ during $\mathrm{RG}$ flow between two bulk critical points. Rather than tackle the problem in its full generality, we suppose the bulk flow is induced by a slightly relevant bulk perturbation and the new fixed point is near the old one. Thus we may use conformal perturbation theory to calculate the change in $g$. It was a similar analysis which motivated the original $g$ theorem conjecture by Affleck and Ludwig [2]. This method was also used in perturbative proofs of the $c$ theorem [9] and the $g$ theorem [3].

There are two good reasons to take this approach. First, it avoids the problem of finding an appropriate off-critical definition of $g$, because one is always computing $g$ at a fixed 
point. Some previous work introduced a particular off-critical definition that is amenable to calculation; however, this might not be the best criteria to use [10, 11]. Second, our result will depend only on general features of the conformal field theory. Previous work on bulk flow in theories with boundaries has largely focused on specific models «10, 11, 12, 13, 14, 15, 16, 17, 18]. Because each model has very different properties, it is difficult to identify the general features. For example, the bulk flow studied in [10, 11, 16] focused on the non-unitary Lee-Yang model. Features of these flows could be attributed to the lack of unitarity, rather than being generic to bulk flows.

We begin in section [I] with a brief overview of $g$. Next, in section III, we calculate the change in $g$ under bulk RG flow. We find that the change in $g$ does not have a definite sign, and so it can increase during certain bulk flows. In section IV we demonstrate this behavior explicitly for certain flows between minimal models. We then go on in section $\nabla$ to discuss various applications of this result.

\section{OVERVIEW OF $\mathrm{g}$}

Consider a one-dimensional quantum system of length $L$ at a temperature $T=1 / \beta$, with boundary conditions $A$ and $B$ at the two ends. Near criticality, the free energy $\log Z$ takes the form [19, 20]

$$
\log Z=\log \operatorname{Tr} e^{-\beta H_{A B}}=\log g+\frac{c \pi}{6 \beta} L-\beta E_{0},
$$

so long as the size of the system $L \gg \beta$ is large ${ }^{1}$. Here $H_{A B}$ is the Hamiltonian for the system with the prescribed boundary conditions, $c$ is the central charge of the associated $\mathrm{CFT}$, and $E_{0}$ is the ground-state energy of $H_{A B}$.

The first two terms in (11) are universal and determined by conformal invariance: they depend only on properties of the nearby critical point. The third term, $\beta E_{0}$, is a nonuniversal piece: it is sensitive to the details of the theory. It is clear from this expression that $\log (g)$ is the entropy of the system at zero temperature. For systems with finite size $L$ the spectrum is discrete and $g$ is an integer, the ground-state degeneracy $\left(Z \sim g e^{-\beta E_{0}}\right)$. As $L$ increases the spectrum becomes continuous, and $g$ is no longer constrained to integer values.

\footnotetext{
${ }^{1}$ We have suppressed the nonuniversal $\mathcal{O}\left(T^{2}\right)$ and higher-order finite- $T$ corrections in (1).
} 
Alternatively, by interchanging the roles of space and time we may view the boundary conditions $A$ and $B$ as specifying initial and final boundary states $|A\rangle$ and $|B\rangle$, between which the system propagates for a time $L$ with periodic boundary conditions on a spatial circle of circumference $\beta[21,22]$. From this perspective the partition function may be written

$$
Z=\left\langle B\left|e^{-L H_{P}}\right| A\right\rangle,
$$

where $H_{P}$ is the Hamiltonian of the spatially periodic system. In the limit $L / \beta \rightarrow \infty$ this becomes

$$
Z \sim\langle A \mid 0\rangle e^{-L E_{0}}\langle 0 \mid B\rangle
$$

We thus identify $g \equiv g_{A} g_{B}=\langle A \mid 0\rangle\langle 0 \mid B\rangle$ and note that $g$ receives multiplicative contributions from each of the boundaries, as one would expect (the corresponding contributions to the entropy are additive).

\section{PERTURBATIVE EVALUATION OF $\mathrm{g}$}

In this section we calculate how $g$ changes under bulk RG flow. First, however, we discuss some general considerations and summarize relevant previous results on perturbative flow between critical points. Then we explicitly calculate the leading change in $g$. Finally, we discuss the more general flow.

\section{A. General Considerations and Summary of Previous Results}

Let $S_{\mathrm{BCFT}}$ be the action for our critical BCFT. In general, one may perturb this theory by some combination of relevant bulk operators $\Phi_{i}(z, \bar{z})$ and relevant boundary operators $\Psi_{j}(x)$. The resulting theory is described by the action $S$,

$$
S=S_{\mathrm{BCFT}}+\sum_{i} \lambda_{i} \int \Phi_{i}(z, \bar{z}) d^{2} z+\sum_{j} \mu_{j} \int \Psi_{j}(x) d x,
$$

and is no longer critical, except perhaps for special values of the couplings $\lambda_{i}, \mu_{j}$. We can now, at least in principle, follow the RG flow induced by this perturbation to the nearest RG fixed point, a theory described by some action $S^{*}$. If the theory described by $S^{*}$ is close to the original BCFT then the resulting flow can be described as a flow in the space

of couplings, and one might hope to understand the system using perturbation theory. If 
the perturbing operators $\Phi_{i}$ and $\Psi_{j}$ have certain nonzero operator products with other bulk or boundary operators $\mathcal{O}_{k}$ in the theory, then the perturbation (4) will induce the flow of those operators $\mathcal{O}_{k}$, as well. (Boundary operators do not induce the flow of bulk operators, however.)

If the flow from $S_{\mathrm{BCFT}}$ to $S^{*}$ involves only boundary operators, then the endpoint $S^{*}$ describes the same bulk theory but with different conformally invariant boundary conditions. If the flow is induced by a bulk operator then the endpoint will be a new bulk theory, with conformally invariant boundary conditions consistent with the new fixed point. The conformal boundary conditions of $S^{*}$ may not have any obvious interpretation in terms of the boundary conditions of $S_{\mathrm{BCFT}}$. This is not an issue in perturbation theory, however, as one only prescribes boundary conditions at the original fixed point.

Rather than study the general case, we make two simplifying assumptions. First, we assume that the fixed point $S^{*}$ is close enough to $S_{\mathrm{BCFT}}$ that perturbation theory is valid throughout the flow. Second, we perturb by only one relevant bulk operator $\Phi$, and we assume that the operator products of this operator with the other bulk and boundary operators $\mathcal{O}_{k}$ are such that none of their flows are induced by the $\Phi$ perturbation. With this second assumption, we can ensure the validity of perturbation theory by choosing the operator $\Phi$ to be only slightly relevant.

We now recall some important results towards understanding flows between conformal field theories. For CFTs without boundary there is the famous Zamolodchikov $c$-theorem [1]. This theorem states that the central charges $c$ and $c^{*}$ of two fixed points $S_{\mathrm{CFT}}$ and $S_{\mathrm{CFT}}^{*}$ must satisfy $c>c^{*}$ if one can flow from $S_{\mathrm{CFT}}$ to $S_{\mathrm{CFT}}^{*}$ along an RG trajectory. To prove this theorem Zamolodchikov generalized the definition of central charge to nonconformal theories by introducing the so-called $c$-function. For a conformal field theory, the $c$-function agrees with the central charge. Zamolodchikov was able to show that the $c$-function decreases along RG flow, so the central charges must decrease.

Cardy and Ludwig [9] addressed this same question in the regime of perturbation theory. They perturbed a theory $S_{\mathrm{CFT}}$ by a slightly relevant operator with conformal weights $h=$ $\bar{h}=1-y$, where $0<y \ll 1$, and used perturbative techniques to investigate the properties of the endpoint $S_{\mathrm{CFT}}^{*}$. Among other things, they found the leading-order change $\delta c=c^{*}-c$ in the central charge to be $\delta c=-y^{3} / b^{2}$, where $b$ is the coefficient in the three-point function of the canonically normalized perturbing operator. Because $\delta c$ has a definite sign, this shows 
that $c$ decreases in perturbation theory.

For CFTs with boundary the situation is more complicated, because one must keep track of both the bulk theory and the boundary conditions. Previous research has focused on purely boundary flows. Affleck and Ludwig [2] first studied this situation in the context of the Kondo effect [3, 8]. They originally introduced the quantity $g$ and understood its physical interpretation. They studied the change in $g$ perturbatively and found that, under flow generated by a slightly relevant boundary operator of conformal weight $h=1-y$, with $0<y \ll 1, g$ changes by $\delta g=-\pi^{2} y^{3} g / 3 b^{2}$ (again, $b$ is related to the three-point function of the perturbing boundary operator), and so $g$ decreases in perturbation theory. Friedan and Konechny [4] have given a non-perturbative proof of the $g$ theorem.

Assuming $b$ is $\mathcal{O}(1)$, we can summarize these findings by saying that, for bulk flow of a boundary-less CFT, $\delta c \sim-\mathcal{O}\left(y^{3}\right)$, and for purely boundary flow of a BCFT, $\delta g \sim-\mathcal{O}\left(y^{3}\right)$. The quantities $c$ and $g$ decrease under these flows. This leaves open the question of how $g$ changes when the bulk flows between critical points, which is the subject of this paper.

\section{B. Leading-Order Calculation}

Consider a boundary conformal field theory with action $S_{\mathrm{BCFT}}$ that contains a bulk primary operator $\Phi(z, \bar{z})$ with conformal weights $h=\bar{h}=1-y$, where $0<y \ll 1$. The scaling dimension $x$ of $\Phi(z, \bar{z})$ is thus $x=h+\bar{h}=2-2 y$. If we perturb the action $S_{\mathrm{BCFT}}$ by this operator then the resulting theory is no longer critical, and it has an action $S$ given by

$$
S=S_{\mathrm{BCFT}}-a^{-2 y} \lambda \int d^{2} z \Phi(z, \bar{z}) .
$$

Here $\lambda$ is the bare coupling and $a$ is a short distance cutoff required to make $\lambda$ dimensionless.

Under RG flow the renormalized coupling $\lambda(\ell)$ runs according to the $\beta$-function equation

$$
\frac{d \lambda(\ell)}{d \log (\ell)}=\beta(\lambda)=2 y \lambda-\pi b \lambda^{2}+\cdots,
$$

and so there are fixed points at $\lambda=0$ and $\lambda=\lambda^{*} \equiv 2 y / \pi b$. We take $\ell$ to be the length scale at which the theory is defined. The constant $b$, which we take to be $\mathcal{O}(1)$, is related to the 
coefficient of the bulk three-point function of $\Phi:^{2}$

$$
\left\langle\Phi\left(z_{1}, \bar{z}_{1}\right) \Phi\left(z_{2}, \bar{z}_{2}\right) \Phi\left(z_{3}, \bar{z}_{3}\right)\right\rangle=\frac{-b}{\left|z_{12}\right|^{x}\left|z_{23}\right|^{x}\left|z_{31}\right|^{x}} .
$$

Note that in writing (6) we have assumed that the $\Phi(z, \bar{z})$ perturbation does not induce the flow of any other relevant bulk operators. Furthermore, we will assume that no boundary flows are induced. We relax these assumptions and discuss the more general flow in the next section.

We wish to calculate the change in $g$ between the two fixed points, when $\lambda^{*} \sim y \ll 1$ is sufficiently small that perturbation theory is valid throughout the flow. In general one must regulate a perturbative calculation by considering a finite-size system at non-zero temperature. We encounter no divergences with length, so we are free to put our system on an infinite half-cylinder. The inverse temperature is then encoded in the circumference $\beta$, and the single conformal boundary condition we label by $k$. (We shall therefore be studying the boundary entropy $g_{k}$ associated to $k$.)

In order to compute the change $\delta g / g$, consider

$$
\log Z-\log Z_{0}=\frac{Z}{Z_{0}}-1+\mathcal{O}\left(\lambda^{2}\right)=a^{-2 y} \lambda \int d^{2} w\langle\Phi(w, \bar{w})\rangle_{\mathrm{CYL}}+\cdots
$$

where $Z\left(Z_{0}\right)$ is the partition function of the (un)perturbed system. Note that the leadingorder correction in (8), which we shall hereafter denote by $Z_{1}$, involves the one-point function of the bulk operator $\Phi$. If our theory were on a manifold without boundary then the operator $\Phi$ could be chosen to have vanishing one-point function, and then the leading correction would be given by a two-point function. Similarly, if we were to perturb our BCFT by a boundary operator, then the one-point function of that operator along the boundary could be chosen to vanish. But the one-point function of a bulk operator on a manifold with boundary does not vanish in general, and, in fact, it depends on the boundary conditions.

From (11) we see that $Z_{1}$ could contain three contributions: the term $\log (1+\delta g / g) \approx \delta g / g$ (to lowest order) that interests us, a piece that depends on the central charge, and a groundstate energy correction. To the order that we are working the central charges of the two fixed points $S_{\mathrm{BCFT}}$ and $S^{*}$ agree, and so the corresponding corrections will not appear in our

\footnotetext{
${ }^{2} \beta(\lambda)$ is determined by the bulk theory alone, so this three-point correlation function is computed in the theory without boundary. We normalize $\Phi$ to have the standard two-point function $\left\langle\Phi\left(z_{1}, \bar{z}_{1}\right) \Phi\left(z_{2}, \bar{z}_{2}\right)\right\rangle=$ $\left|z_{1}-z_{2}\right|^{-x}$.
} 
computation. Thus to isolate the term $\delta g / g$ we need only identify and discard the groundstate energy renormalizations. This is easy because such contributions scale linearly with the inverse temperature $\beta$.

The one-point function on the half cylinder can be computed by conformal transformation from the familiar result for the upper half plane. The one-point function on the upper half plane with standard coordinate $z$ is constrained by conformal invariance to be of the form

$$
\langle\Phi(z, \bar{z})\rangle_{\mathrm{UHP}}=\frac{A_{\Phi}^{k}}{|2 \operatorname{Im} z|^{x}}
$$

where $A_{\Phi}^{k}$ depends on both the particular field $\Phi$ and the choice of conformal boundary condition $k$. Because the coefficient $A_{\Phi}^{k}$ has no definite sign, we will see that $g$ can increase during bulk RG flow.

Let us coordinatize the cylinder by $w=\tau+i \sigma$; the imaginary direction $\sigma$ winds around the compact circle, and the real direction $\tau$ measures distance from the boundary. Then a suitable conformal mapping to the half plane is $z=i \tanh (\pi w / \beta)$. We can now compute

$$
\langle\Phi(w, \bar{w})\rangle_{\mathrm{CYL}}=\left|\frac{d z}{d w}\right|^{x}\langle\Phi(z, \bar{z})\rangle_{\mathrm{UHP}}=\frac{A_{\Phi}^{k}}{\left(\frac{\beta}{\pi} \sinh \left(\frac{2 \pi}{\beta} \tau\right)\right)^{x}} .
$$

The leading contribution $Z_{1}$ is thus

$$
Z_{1}=a^{-2 y} \lambda \int_{0}^{\beta} d \sigma \int_{a}^{\infty} d \tau \frac{A_{\Phi}^{k}}{\left(\frac{\beta}{\pi} \sinh \left(\frac{2 \pi}{\beta} \tau\right)\right)^{2-2 y}}
$$

After performing the integral over the compact $\sigma$ coordinate and changing variables to $r=\exp (4 \pi \tau / \beta)$ we are left with

$$
Z_{1}=\pi \lambda A_{\Phi}^{k}\left(\frac{\beta}{2 \pi a}\right)^{2 y} \int_{a^{\prime}}^{\infty} \frac{d r}{r^{y}(r-1)^{2-2 y}}
$$

where $a^{\prime}=e^{\frac{4 \pi a}{\beta}}$. The integral diverges in the limit $a^{\prime} \rightarrow 1$ of small cutoff $(a \rightarrow 0)$. In order to understand the nature of this divergence we integrate by parts to extract it from the integral.

Integrating by parts once gives

$$
Z_{1}=-\pi \lambda A_{\Phi}^{k} \frac{1}{1-2 y}\left(\frac{\beta}{2 \pi a}\right)^{2 y}\left[\left.\frac{1}{r^{y}(r-1)^{1-2 y}}\right|_{a^{\prime}} ^{\infty}+y \int_{a^{\prime}}^{\infty} \frac{d r}{r^{1+y}(r-1)^{1-2 y}}\right] .
$$

The integral in (13) is now convergent as $a^{\prime} \rightarrow 1$. However, it diverges at $y=0$, and our ultimate goal is a perturbative calculation in $y$. To remedy this we integrate by parts a 
second time:

$$
Z_{1}=-\pi \lambda A_{\Phi}^{k} \frac{1}{1-2 y}\left(\frac{\beta}{2 \pi a}\right)^{2 y}\left[\left.\frac{(r-1)^{2 y}}{r^{y}} \frac{3 r-1}{2 r(r-1)}\right|_{a^{\prime}} ^{\infty}+\frac{1+y}{2} \int_{a^{\prime}}^{\infty} \frac{(r-1)^{2 y}}{r^{2+y}} d r\right] .
$$

The prefactor $\lambda(\beta / a)^{2 y}$ will eventually be expressed in terms of the renormalized coupling, so for now we concentrate on the $y$ dependence inside the brackets. The integral in (14) is convergent as $a^{\prime} \rightarrow 1$ and $y \rightarrow 0$, so we may expand it in powers of $y$. To lowest order, we find

$$
Z_{1}=\pi \lambda A_{\Phi}^{k}\left(\frac{\beta}{2 \pi a}\right)^{2 y}\left[\left(\frac{\beta}{4 \pi a}\right)^{1-2 y}-\frac{1}{2}+\mathcal{O}\left(y, \frac{a}{\beta}\right)\right] .
$$

The first term is simply a ground-state energy correction; this we discard in our calculation of $\delta \mathrm{g} / \mathrm{g}$. The second term is the leading contribution to the change in $g$.

In order to determine how $g$ changes between the two fixed points we must rewrite $\delta g / g$ in terms of the renormalized coupling $\lambda(\beta)$ (we take $\beta$ to be the length scale $\ell$ at which the coupling is defined). The solution to the RG equation (6) is

$$
\lambda\left(\frac{\beta}{a}\right)^{2 y}=\frac{\lambda(\beta)}{1-\frac{\lambda(\beta)}{\lambda^{*}}\left(1-\left(\frac{a}{\beta}\right)^{2 y}\right)},
$$

where $\lambda=\lambda(a)$ is the coupling evaluated at the UV cutoff. In the regime $\lambda(\beta) \ll \lambda^{*}$ we can approximate $\lambda(\beta) \approx \lambda(\beta / a)^{2 y}$ and write $\delta g / g$ in terms of the renormalized coupling $\lambda(\beta)$ :

$$
\frac{\delta g}{g}=-\frac{\pi}{2} \lambda(\beta) A_{\Phi}^{k}+\mathcal{O}\left(y^{2}\right)
$$

So long as the new fixed point $\lambda^{*} \ll 1$ is close to zero and perturbation theory is valid, this last expression should remain true throughout the entire flow. In particular, it will remain true in the low-temperature limit $\beta \rightarrow \infty$, as the coupling approaches the new critical point $\lambda^{*}=2 y / \pi b$.

We find

$$
\frac{\delta g}{g}=-\frac{y}{b} A_{\Phi}^{k}+\mathcal{O}\left(y^{2}\right)
$$

Thus $g$ can either increase or decrease, depending upon the relative signs of $A_{\Phi}^{k}$ and $b$. Note that the sign of $A_{\Phi}^{k}$ depends on both the choice of bulk operator $\Phi$ and the choice of boundary condition $k$; it is not determined by the bulk theory. 


\section{Induced Flows and Higher-Order Terms}

In our leading-order calculation we assumed that the $\Phi$ perturbation did not induce the flow of any other operators. However, bulk-induced boundary operators appear frequently [17, 23, 24, 25], and in many cases they are the dominant effect. These flows generally reduce $g$ to the lowest available value. The bulk perturbation, meanwhile, may increase $g$ for some boundary conditions and decrease it for others.

Suppose the bulk perturbation (5) induces the flow of a boundary operator $\Psi$ of scaling dimension $1-x$, with $x \ll 1$. The $\beta$-function for the coupling $\mu$ of the operator $\Psi$ is $[23]$

$$
\beta(\mu)=x \mu+B_{\Phi \Psi} \lambda-b_{\Psi} \mu^{2}+\ldots,
$$

where $B_{\phi \psi}$ is the coefficient of the bulk-boundary OPE of $\Phi$ and $\Psi$, and $b_{\Psi}$ is the coefficient of the boundary three-point function. The behavior of $g$ now depends on the relative

magnitudes of $x$ and $y$. For example, if $y^{\frac{1}{3}} \ll x \ll 1$ then the boundary RG flow will dominate, and $g$ will decrease. In regimes where the purely boundary and purely bulk effects are competitive, one would also need to include the contribution from the bulk-boundary correlator.

Above we found that bulk perturbations with $A_{\Phi}^{k}=0$ leave $g$ unchanged to leading order in $y$. To better understand the effects of such perturbations we would need to include higherorder corrections. In cases where there is no induced flow the next correction arises from the bulk two-point function. This is more difficult to address, because the two-point function of a primary operator on a manifold with boundary is only fixed by conformal invariance up to a function (rather than just a constant), which depends upon the specifics of the theory. We did not compute this contribution.

\section{Marginal Operators}

Exactly marginal operators describe a continuous family of conformal field theories, labeled by the coupling of the operator. Understanding the space of such theories is different from understanding the behavior of a single theory under RG flow. Nevertheless, the exactly marginal case can be viewed as a limit of RG flow between nearby fixed points. In this spirit we may adapt the above analysis to the exactly marginal case. 
We shall again assume that the deformation induces no boundary flows. This requires the existence of a continuous family of conformal boundary conditions, also labeled by the coupling. If no such family of boundary conditions exists then the deformation will necessarily induce boundary flow. For a more detailed study of exactly marginal operators, see [23, 24, 25].

To understand the behavior of $g$ in the marginal case we simply evaluate (11) at $y=0$. The calculation is easier than before, as the integral can be worked out explicitly. The result is

$$
Z_{1}=\frac{\pi}{2} \lambda A_{\Phi}^{k}\left(\frac{\beta}{4 \pi a}-1\right)+\mathcal{O}\left(\frac{a}{\beta}\right)
$$

As before, the first term is a correction to the ground state energy, and the second term is the contribution to $\delta g / g$. So, for an exactly marginal deformation,

$$
\frac{\delta g}{g}=-\frac{\pi}{2} \lambda A_{\Phi}^{k}+\mathcal{O}\left(\lambda^{2}\right)
$$

Evidently, marginal deformations can also serve to either increase or decrease $g$.

A peculiar application of this was already known from [26, 27], where it was found that one can reverse the effects of a relevant boundary flow by a series of marginal deformations. The physics involved is simply that of a free boson on a circle. The relevant boundary flow in question changes the boundary conditions from Neumann to Dirichlet. However, if the size of the circle is at the self-dual point then there exists a marginal boundary deformation that changes the boundary conditions back to Neumann from Dirichlet. Furthermore, the bulk theory contains an exactly marginal operator that changes the size of the circle. It is now clear how to reverse the effects of the initial relevant boundary flow. Simply deform the size of the circle to the self-dual point, return the boundary conditions to Neumann, and then restore the circle to its original size. For Neumann boundary conditions $g$ is proportional to the radius of the circle, so during this procedure the marginal bulk deformation increases the value of $g$.

\section{MINIMAL MODELS}

Now we provide a class of examples in which $g$ increases during bulk RG flow. The BCFTs of interest are the minimal models with boundary. 
The minimal models are the unitary rational conformal field theories with central charge $c=1-\frac{6}{m(m+1)}<1$, where $m \geq 3$. Their (primary) operator content is parameterized by two integers $(r, s)$, with $1 \leq r \leq m-1$ and $1 \leq s \leq m$, modulo the conformal grid symmetry $(r, s) \sim(m-r, m+1-s)$. The conformal weights of the operator $\Phi_{(r, s)}$ are

$$
h_{m}(r, s)=\bar{h}_{m}(r, s)=\frac{((m+1) r-m s)^{2}-1}{4 m(m+1)} .
$$

For a BCFT there is a one-to-one correspondence between primary bulk operators and conformally invariant boundary conditions.

We are interested in a specific class of flows, first understood perturbatively by Zamolodchikov [28]. When a minimal model at level $m$ is deformed by its least relevant operator, $\Phi_{(1,3)}$, the theory flows to the level $m-1$ minimal model. In the limit of large $m$ these theories cluster near $c=1$, and these RG flows can be understood using a perturbative expansion in $m^{-1}$. Note that $\delta c \sim 1 / m^{3}$ between nearby minimal models, consistent with the assumptions of our general calculation in section 【II.

We will apply our perturbative result (18) to estimate the resulting change in $g$. However, our previous result was derived under the assumption that the bulk perturbation does not induce any boundary flows. We were unable to calculate the general bulk-boundary correlator, and so this is an assumption that we could not explicitly verify. Nevertheless, perturbative boundary flows would contribute only subleading corrections, so we need only worry about large boundary flows that make $\mathcal{O}(1)$ contributions. The minimal models are so simple that one can explicitly calculate $g$ for a given choice of boundary conditions. We find agreement between our perturbative calculation of $\delta g$ and the exact result (to leading order) when the change in $g$ is small enough that it can be understood in perturbation theory. We shall return to this point below.

First we review some useful facts about boundary conditions in minimal models. In section II we saw that a boundary condition can be thought of as specifying a boundary state $|B\rangle$. A particularly simple basis of such states was discovered by Cardy and Lewellen [29], and the basis states $\left|B_{(r, s)}\right\rangle$ are known as Cardy states. They carry a label $(r, s)$ just like the primary operators. These boundary states $\left|B_{(r, s)}\right\rangle$ have the property that their overlap $\left\langle\Phi_{(a, b)} \mid B_{(r, s)}\right\rangle$ with the state $\left|\Phi_{(a, b)}\right\rangle$ (that corresponds to the primary operator 
$\Phi_{(a, b)}$ in the standard state-operator correspondence) is given by

$$
\left\langle\Phi_{(a, b)} \mid B_{(r, s)}\right\rangle=\frac{S_{(r, s)}^{(a, b)}}{\sqrt{S_{(1,1)}^{(a, b)}}}
$$

where $S$ is the modular S-matrix

$$
S_{(r, s)\left(r^{\prime}, s^{\prime}\right)}=(-1)^{1+r s^{\prime}+s r^{\prime}} \sqrt{\frac{8}{m(m+1)}} \sin \left[\pi\left(\frac{m+1}{m}\right) r r^{\prime}\right] \sin \left[\pi\left(\frac{m}{m+1}\right) s s^{\prime}\right] .
$$

From the expression (23) we may compute all of the quantities that are of interest to us. For example, we saw in section \that the boundary entropy $g_{(r, s)}$ of the boundary condition $(r, s)$ is given by $\left\langle 0 \mid B_{(r, s)}\right\rangle$. For unitary CFTs the vacuum state $|0\rangle$ corresponds to the identity operator $1=\Phi_{(1,1)}$, so we find

$$
g_{(r, s)}=\left\langle\Phi_{(1,1)} \mid B_{(r, s)}\right\rangle .
$$

Similarly, the properly normalized one-point function of the operator $\Phi_{(a, b)}$ on the upper half plane with boundary condition $(r, s)$ can be computed in terms of boundary states. In the notation of (9) it is

$$
A_{(a, b)}^{(r, s)}=\frac{\left\langle\Phi_{(a, b)} \mid B_{(r, s)}\right\rangle}{\left\langle 0 \mid B_{(r, s)}\right\rangle} .
$$

We can now begin to understand the flows induced by the $\Phi_{(1,3)}$ operator. The conformal weights of $\Phi_{(1,3)}$ are given by (22). To leading order in $m^{-1}$ this is $h=\bar{h}=1-2 / m$, so we have $y=2 / m$. The coefficient $A_{(1,3)}^{(r, s)}$ of the one-point function is given in (26). To leading order in $m^{-1}$, and assuming $r, s \ll m, A_{(1,3)}^{(r, s)}=\sqrt{3}$. The three-point correlation function was calculated in [30], and to leading order the coefficient is $b=-4 / \sqrt{3}$. Putting all this together in (18) yields

$$
\frac{\delta g}{g}=-\frac{y}{b} A_{(1,3)}^{(r, s)}=\frac{3}{2 m} .
$$

This is positive, and so we conclude that $g$ always increases in the regime $r, s \ll m$. Although (27) was derived under the assumption of no induced boundary flow, any perturbative boundary flow would contribute a subleading correction at order $\mathcal{O}\left(\mathrm{m}^{-3}\right)$.

To understand the matter more fully, we use our knowledge of minimal models to calculate the change in $g$ when the initial theory at level $m$ has Cardy boundary condition $(r, s)$ and the final theory at level $m-1$ has $\left(r^{*}, s^{*}\right)$. Using (25) we find

$$
g_{(r, s)}(m)=\left[\frac{8}{m(m+1)}\right]^{\frac{1}{4}} \frac{\sin \left(\frac{\pi r}{m}\right) \sin \left(\frac{\pi s}{m+1}\right)}{\sqrt{\sin \left(\frac{\pi}{m}\right) \sin \left(\frac{\pi}{m+1}\right)}} .
$$


Expanding to $\mathcal{O}\left(m^{-1}\right)$, and assuming $r, s \ll m$, gives

$$
\frac{\delta g}{g}=\log \left[\frac{g_{(r, s)}(m-1)}{g_{\left(r^{*}, s^{*}\right)}(m)}\right]=\log \left(\frac{r s}{r^{*} s^{*}}\right)+\frac{3}{2 m} .
$$

We see that the change $\delta g$ contains two pieces: an order $\mathcal{O}(1)$ logarithm and an $\mathcal{O}\left(m^{-1}\right)$ correction. The $\mathcal{O}\left(\mathrm{m}^{-1}\right)$ perturbation agrees with our calculation (27).

Equations (27) and (29) provide a constraint on the allowed perturbative flows between nearby boundary minimal models. Specifically, given an initial boundary Cardy state $(r, s)$, an endpoint Cardy state $\left(r^{*}, s^{*}\right)$ must satisfy $r s=r^{*} s^{*}$. If the endpoint boundary condition is instead a linear combination of Cardy states, then cancellations must occur to agree with the perturbative result (27). Under the reasonable assumption that the boundary overlaps with the Cardy states are non-negative integers, such a linear combination must in fact be a pure Cardy state.

The agreement betweeen (27) and (29) is somewhat remarkable. A similar analysis, for purely boundary flows in minimal models, was performed in [31]. There it was shown that this simple type of analysis fails for most boundary conditions. In particular, matching the perturbative and exact results could not be accomplished with a simple flow between Cardy states, but rather required the end point to be some linear combination. The agreement of our results for the Cardy states satisfying $r^{*} s^{*}=r s$ is consistent with the existence of such flows, which would increase $g$.

One difficulty in understanding boundary flows lies in the fact that the initial and final bulk theories are different, so boundary conditions labeled by the same integer pairs will have different meaning before and after the bulk RG flow. Thus it is not clear what endpoint boundary condition results from a given initial boundary condition, even when there is no induced boundary flow. More prosaically, the endpoint $m-1$ minimal model contains fewer primary operators than the initial $m$ theory, and so it admits correspondingly fewer conformally invariant boundary conditions.

We suspect that the flow from $(1,1)$ to $\left(1^{*}, 1^{*}\right)$ exists. As mentioned above, Zamolodchikov showed [28] that the $\Phi_{(1,3)}$ operator induces flow between nearby minimal models. This should remain true in the presence of a boundary so long as the initial boundary conditions can smoothly flow into some boundary condition of the resulting theory. The $(1,1)$ boundary state has the lowest $g$ among Cardy states, so it is unlikely that it induces boundary flow. Furthermore, it is known that, in the bulk flow induced by $\Phi_{(1,3)}$, the primary operators 
$\Phi_{n n}$ of the $m$ theory flow to the $\Phi_{n n}$ operators of the $m-1$ theory [28]. Thus, noting the one-to-one correspondence between boundary states and bulk primary operators, it seems reasonable that the Cardy state $(1,1)$ should flow to the corresponding boundary condition in the $m-1$ theory.

\section{INTERPRETATIONS AND APPLICATIONS}

\section{A. String Theory}

Renormalization group flow in the bulk of a two-dimensional field theory is often used to understand closed string tachyon condensation. In this context the relevant operator is the vertex operator for a negative-mass state in the spacetime description.

RG flow of the worldsheet field theory can approximate time evolution of the target space theory [32, 33, 34, 35], but in general the two are different. In particular, the RG flow yields first-order equations of motion, while the time evolution is governed by secondorder equations. RG flow becomes a good approximation in supercritical theories with large, negative dilaton time derivations [32, 33, 34, 35]. In such cases the friction term dominates the time evolution, yielding approximately first-order behavior (much like slow-roll inflation).

In other cases RG flow yields the same endpoint as time evolution. This happens for tachyon condensation processes that are localized in space, where energy density can escape to infinity. With decays to "nothing," where entire regions of spacetime disappear, RG and time evolution seem to give qualitatively similar results. In these scenarios all of the closed string states become exponentially massive (including the graviton), and it is in this way that the spacetime description is lost. Here the agreement between the two approaches might be due to the graviton mass, which impedes backreaction.

The inclusion of boundaries on the worldsheet now describes a spacetime containing Dbranes. The tension of these branes is given by $g$ [6]. The behavior of D-branes under closed string tachyon condensation is potentially very interesting, as they may react to the condensate in a qualitatively differently way from the usual string states. If they survive into regions where string states become lifted, then this might signal a breakdown in the unitarity of the spacetime theory. Just this phenomenon has been argued to occur in the $c=1$ matrix model [36]. While the underlying theory remains unitary, it is still disturbing 
to lose unitarity in spacetime.

In other contexts [17, 18] it has been shown that this does not occur: the branes gain exponentially large masses $(g \rightarrow \infty)$ [17] or completely decouple $(g \rightarrow 0)$ [18]. Both would be consistent with our expectations from the closed string sector. The decoupling case is similar to open string tachyon condensation processes, where the brane disappears from the spacetime description. This is distinguished from the D-brane simply becoming light by the absence of couplings to any closed string modes (term-wise vanishing of the open-string partition function, as opposed to cancellations between separate contributions). To preserve spacetime unitarity one would prefer the branes to become massive or decouple, rather than persist unaffected.

Our results suggest that the brane tension is sensitive to the tachyon condensation even if the tachyon does not modify the boundary conditions. Closed string tachyon condensation alone can cause the brane to become extremely massive. We have not shown that this is the generic outcome (although it is suggested in [17]). However, the sensitivity of the tension to the bulk RG makes it unlikely for the brane to be unaffected by a closed string tachyon condensate.

One further motivation for this work was to resolve some confusions associated with [17]. The definition of "mass" used there is not equivalent to $g$, so it was unclear whether $g$ should increase along these flows. This work is consistent with the interpretation that the branes do have growing masses, as the results in both cases depend on the sign of $A_{\Phi}^{k}$ in the same way.

Of course, string theory is more than just CFT on a disk; it includes a sum over all metrics and topologies of the worldsheet, as well. Taking this into account, [37] determined the backreaction of a brane on the spacetime geometry. The leading effect, due to the Fischler-Susskind mechanism, is a modification of the beta function for the bulk coupling:

$$
\frac{d \lambda}{d \log (\ell)}=\beta(\lambda)=2 y \lambda+g_{s} \frac{g}{\pi} A_{\Phi}^{k}-\pi b \lambda^{2},
$$

where $g_{s}$ is the string coupling. If one can stabilize the dilaton such that $g_{s} \ll y^{2} / b g A_{\Phi}^{k}$ then our perturbative analysis holds, though with a slighly modified fixed point, yielding

$$
\frac{\delta g}{g}=-\frac{y}{b} A_{\Phi}^{k}-g_{s} \frac{g}{4 y}\left(A_{\Phi}^{k}\right)^{2}+\mathcal{O}\left(y^{2}\right)
$$

We see that the backreaction always decreases $g$, in this limit. This is consistent with the 
observations [17, 18, 37] that induced boundary flows and backreactions tend to minimize the mass of branes.

\section{B. Condensed Matter}

Two-dimensional boundary conformal field theories have been used to understand the multi-channel Kondo effect [3, 38, 39] (see [8] for a review). In fact, it is in this context that Affleck and Ludwig originally defined $g$ [2]. Here one is trying to understand the role of impurities that are coupled via a spin interaction to a system of free fermions. The problem is reduced to $1+1$ dimensions by concentrating on $s$-wave or radial scattering of the electrons from the impurity. In the UV, the coupling of the impurity to the electrons acts as a relevant boundary operator perturbing the free fermion BCFT. In the IR, at the strongly-coupled fixed point, the theory is described by free fermions with a new, nontrivial boundary condition. This approach is particularly powerful in the over-screened case, where the appropriate boundary condition has no simple description in terms of free fermions. Nevertheless, one can deduce the correct boundary theory from the fusion rules.

In this context $\log (g)$ is the impurity entropy. When there is a suitable free fermion description (the critical and under-screened cases), $g$ agrees with the ground-state degeneracy, which can be determined independently. In all cases $g$ is lower at the strong-coupling fixed point (IR) than at the free fixed point (UV). This follows directly from the $g$ theorem.

In this language our result is simply a statement about the effects that bulk interactions can have on the impurity entropy. While the free fermion description might be a good approximation for some systems, this is not the case in general. Our results show that the impurity entropy can increase or decrease as we lower the temperature of the system, due only to interactions between the fermions.

Further applications would be interesting. Recently [40], a connection has been made between the boundary entropy and the universal part of the entanglement entropy [41, 42] of a system exhibiting a topological phase. Both quantities are related to a particular entry of the modular S-matrix of an associated CFT (for further elaboration, please see [43]). We would like to make more direct contact between $g$ and the physics of topological phases. 


\section{CONCLUSION}

We have studied the effect of bulk RG flow on the boundary entropy of a two-dimensional BCFT when the flow is due to perturbation by a single (slightly) relevant primary operator of scaling dimension $2-2 y$. To leading order the change in $g$ was found to be $\delta g / g=-A y / b$, where $A$ is the coefficient of the one-point function and $b$ the coefficient of the three-point function. This expression has no preferred sign, and so $g$ can either increase or decrease, depending upon the choice of perturbing operator and boundary conditions. We saw an explicit realization of this in the minimal models, where the flows induced by the $\Phi_{(1,3)}$ operator can increase $g$ for certain boundary conditions. All of this is in stark contrast to the behavior of $g$ under purely boundary perturbations, for which the $g$ theorem guarantees that $g$ will decrease. Our result has applications to closed string tachyon condensation and the multi-channel Kondo effect.

\section{Acknowledgments}

We would like to thank Eduardo Fradkin, Matt Headrick, Shamit Kachru, Eun-Ah Kim, Albion Lawrence, and Eva Silverstein for helpful discussions. We are supported in part by the DOE under contract DE-AC03-76SF00515 and by the NSF under contract 9870115. DG is also supported by a NSERC Postgraduate fellowship and a Mellam Family Foundation Fellowship.

[1] A. B. Zamolodchikov, JETP Lett. 43, 730 (1986).

[2] I. Affleck and A. W. W. Ludwig, Phys. Rev. Lett. 67, 161 (1991).

[3] I. Affleck and A. W. W. Ludwig, Phys. Rev. B 48, 7297 (1993).

[4] D. Friedan and A. Konechny, Phys. Rev. Lett. 93, 030402 (2004), hep-th/0312197.

[5] J. Polchinski, Phys. Rev. Lett. 75, 4724 (1995), hep-th/9510017.

[6] J. A. Harvey, S. Kachru, G. W. Moore, and E. Silverstein, JHEP 03, 001 (2000), hepth/9909072.

[7] I. Affleck and A. W. W. Ludwig, Phys. Rev. Lett. 68, 1046 (1992).

[8] I. Affleck, Acta Phys. Polon. B26, 1869 (1995), cond-mat/9512099. 
[9] A. W. W. Ludwig and J. L. Cardy, Nucl. Phys. B285, 687 (1987).

[10] P. Dorey, D. Fioravanti, C. Rim, and R. Tateo, Nucl. Phys. B696, 445 (2004), hep-th/0404014.

[11] P. Dorey, A. Lishman, C. Rim, and R. Tateo, Nucl. Phys. B744, 239 (2006), hep-th/0512337.

[12] S. Ghoshal and A. B. Zamolodchikov, Int. J. Mod. Phys. A9, 3841 (1994), hep-th/9306002.

[13] A. LeClair, G. Mussardo, H. Saleur, and S. Skorik, Nucl. Phys. B453, 581 (1995), hepth/9503227.

[14] F. Lesage, H. Saleur, and P. Simonetti, Phys. Lett. B427, 85 (1998), hep-th/9802061.

[15] C.-r. Ahn and C. Rim, J. Phys. A32, 2509 (1999), hep-th/9805101.

[16] P. Dorey, I. Runkel, R. Tateo, and G. Watts, Nucl. Phys. B578, 85 (2000), hep-th/9909216.

[17] D. Green, JHEP 04, 025 (2007), hep-th/0611003.

[18] M. R. Gaberdiel and A. Lawrence, JHEP 05, 087 (2007), hep-th/0702036.

[19] H. W. J. Bloete, J. L. Cardy, and M. P. Nightingale, Phys. Rev. Lett. 56, 742 (1986).

[20] I. Affleck, Phys. Rev. Lett. 56, 746 (1986).

[21] N. Ishibashi, Mod. Phys. Lett. A4, 251 (1989).

[22] J. L. Cardy, Nucl. Phys. B324, 581 (1989).

[23] S. Fredenhagen, M. R. Gaberdiel, and C. A. Keller, J. Phys. A40, F17 (2007), hep-th/0609034.

[24] M. Baumgartl, I. Brunner, and M. R. Gaberdiel, JHEP 07, 061 (2007), arXiv:0704.2666 [hep-th].

[25] S. Fredenhagen, M. R. Gaberdiel, and C. A. Keller (2007), arXiv:0707.2511 [hep-th].

[26] A. Recknagel and V. Schomerus, Nucl. Phys. B545, 233 (1999), hep-th/9811237.

[27] A. Sen, Int. J. Mod. Phys. A14, 4061 (1999), hep-th/9902105.

[28] A. B. Zamolodchikov, Sov. J. Nucl. Phys. 46, 1090 (1987).

[29] J. L. Cardy and D. C. Lewellen, Phys. Lett. B259, 274 (1991).

[30] V. S. Dotsenko and V. A. Fateev, Nucl. Phys. B240, 312 (1984).

[31] A. Recknagel, D. Roggenkamp, and V. Schomerus, Nucl. Phys. B588, 552 (2000), hepth/0003110.

[32] J. Polchinski, Nucl. Phys. B324, 123 (1989).

[33] A. R. Cooper, L. Susskind, and L. Thorlacius, Nucl. Phys. B363, 132 (1991).

[34] C. Schmidhuber and A. A. Tseytlin, Nucl. Phys. B426, 187 (1994), hep-th/9404180.

[35] D. Z. Freedman, M. Headrick, and A. Lawrence, Phys. Rev. D73, 066015 (2006), hepth/0510126. 
[36] J. L. Karczmarek and A. Strominger, JHEP 05, 062 (2004), hep-th/0403169.

[37] C. A. Keller (2007), arXiv:0709.1076 [hep-th].

[38] I. Affleck and A. W. W. Ludwig, Nucl. Phys. B360, 641 (1991).

[39] A. W. W. Ludwig and I. Affleck, Nucl. Phys. B428, 545 (1994).

[40] P. Fendley, M. P. A. Fisher, and C. Nayak, J. Stat. Phys. 126, 1111 (2007), cond-mat/0609072.

[41] A. Kitaev and J. Preskill, Phys. Rev. Lett. 96, 110404 (2006), hep-th/0510092.

[42] M. Levin and X.-G. Wen, Phys. Rev. Lett. 96, 110405 (2006), cond-mat/0510613.

[43] For further elaboration, please see the commented-out discussion in the tex source file. 